\title{
Chambers of 2-affine arrangements and freeness of 3-arrangements
}

\author{
Takuro Abe
}

Received: 11 May 2012 / Accepted: 3 August 2012 / Published online: 24 August 2012

(C) Springer Science+Business Media, LLC 2012

\begin{abstract}
We give a lower bound on the number of chambers divided by a family of lines in the real plane. Also, a sufficient condition for 3-arrangements to be free is given in terms of chambers. To prove them, we give the upper bound on differences of exponents for balanced 2-multiarrangements in terms of the cardinality of hyperplanes.
\end{abstract}

Keywords Hyperplane arrangements · Free arrangements · Chambers of arrangements · Characteristic polynomial $\cdot$ Exponents of multiarrangements

\section{Introduction}

Let $V$ be an $\ell$-dimensional vector space over a field $\mathbb{K}$ of characteristic zero, $S=$ $\mathbb{K}\left[x_{1}, \ldots, x_{\ell}\right]$ the coordinate ring, and $\operatorname{Der}(S)=\oplus_{i=1}^{\ell} S \cdot \partial_{x_{i}}$ the module of $S$-regular derivations. A hyperplane arrangement $\mathcal{A}$ is a finite collection of hyperplanes in $V$. An arrangement $\mathcal{A}$ is called central if every $H \in \mathcal{A}$ is linear. For each $H \in \mathcal{A}$, let us fix a linear form $\alpha_{H} \in V^{*}$ such that $\operatorname{ker} \alpha_{H}=H$. A function $m: \mathcal{A} \rightarrow \mathbb{Z}_{>0}$ is called a multiplicity, and a pair $(\mathcal{A}, m)$ a multiarrangement. Let $|m|:=\sum_{H \in \mathcal{A}} m(H)$. When $m \equiv 1$, a multiarrangement $(\mathcal{A}, 1)$ is the same as an arrangement, which is sometimes called a simple arrangement. An $\ell$-arrangement is that in $V \simeq \mathbb{K}^{\ell}$. An arrangement $\mathcal{A}$ is said to be affine if $\mathcal{A}$ is not necessarily central. When $\mathbb{K}=\mathbb{R}$, an arrangement in $V=\mathbb{R}^{\ell}$ is said to be a real arrangement. In this article, $\mathcal{A}$ often denotes a central arrangement, and $\overline{\mathcal{A}}$ an affine arrangement, unless otherwise specified.

Let us consider the following problem. For a real 2-affine arrangement $\overline{\mathcal{A}}$ defined by equations

$$
\alpha_{i}=k_{i j}\left(\alpha_{i} \in V^{*}, k_{i j} \in \mathbb{R}, i=1, \ldots, h, j=1, \ldots, m_{i}\right)
$$

T. Abe $(\bowtie)$

Department of Mechanical Engineering and Science, Kyoto University, Kyoto 606-8501, Japan e-mail: abe.takuro.4c@kyoto-u.ac.jp 
with $h>2$, how many connected components (called chambers)

$$
\operatorname{ch}(\overline{\mathcal{A}}):=\left\{\text { connected components of } \mathbb{R}^{2} \backslash \cup_{H \in \overline{\mathcal{A}}} H\right\}
$$

can there be? If we can use the whole information of $\overline{\mathcal{A}}$, then the explicit formula for $|\operatorname{ch}(\overline{\mathcal{A}})|$ was obtained by Zaslavsky, see [13] or Theorem 2.1. Next, if we fix the directions of lines $\alpha_{1}, \ldots, \alpha_{h}$ and numbers of lines $m_{i}$ which are parallel to $\alpha_{i}=0$, then can we say something about the minimal number of chambers? We can write down this problem in terms of 2-multiarrangements. Let $\mathcal{A}^{\prime \prime}$ be a central 2-arrangement defined by $\alpha_{i}=0(i=1, \ldots, h)$ and define $m_{0}: \mathcal{A}^{\prime \prime} \rightarrow \mathbb{Z}_{>0}$ by $m_{0}\left(\alpha_{i}=0\right)=m_{i}$. Let us denote this correspondence by $R$, i.e., $R(\overline{\mathcal{A}}):=\left(\mathcal{A}^{\prime \prime}, m_{0}\right)$. Then the problem is to find a lower bound on

$$
\operatorname{MC}\left(\mathcal{A}^{\prime \prime}, m_{0}\right):=\min _{R(\overline{\mathcal{A}})=\left(\mathcal{A}^{\prime \prime}, m_{0}\right)}|\operatorname{ch}(\overline{\mathcal{A}})| .
$$

One of these lower bounds is given by Yoshinaga in [12]. See Theorem 4.1.

Next generalize this problem as follows. In the previous paragraph, we started from a multiarrangement $\left(\mathcal{A}^{\prime \prime}, m\right)$ which knows not only the directions but also the numbers of lines parallel to each direction. In this paragraph, let us start from less data and consider a larger family of arrangements. Namely, we start from the number of lines and directions as follows. First define

$$
N\left(\mathcal{A}^{\prime \prime}, m_{0}\right):=\left(\left|\mathcal{A}^{\prime \prime}\right|,\left|m_{0}\right|\right)=\left(\left|\mathcal{A}^{\prime \prime}\right|,|\overline{\mathcal{A}}|\right)=(h, n)
$$

and

$$
N R(\overline{\mathcal{A}}):=N(R(\overline{\mathcal{A}}))=N\left(\mathcal{A}^{\prime \prime}, m_{0}\right)=(h, n) .
$$

Then we want to know the lower bound on

$$
\operatorname{MC}\left(\left|\mathcal{A}^{\prime \prime}\right|,\left|m_{0}\right|\right):=\min _{N R(\overline{\mathcal{A}})=\left(\left|\mathcal{A}^{\prime \prime}\right|,\left|m_{0}\right|\right)}|\operatorname{ch}(\overline{\mathcal{A}})| .
$$

If some $H \in \mathcal{A}^{\prime \prime}$ satisfies $m_{0}(H)>\sum_{K \in \mathcal{A}^{\prime \prime} \backslash\{H\}} m_{0}(K)$, then the problem is easy, see Remark 4.12 for details. Hence, we consider this problem in the following case. We say that a multiplicity $m_{0}$, or an affine 2 -arrangement $\overline{\mathcal{A}}$, is balanced if

$$
m_{0}(H) \leq \sum_{K \in \mathcal{A}^{\prime \prime} \backslash\{H\}} m_{0}(K) \quad\left(\forall H \in \mathcal{A}^{\prime \prime}\right) .
$$

From now on we consider the problem above only for balanced $\overline{\mathcal{A}}$, unless otherwise specified. Hence, $R$ is a map from the set of balanced affine 2-arrangements to that of balanced 2-multiarrangements. Now we can formulate the problems introduced above in terms of arrangements as follows:

Problem 1.1 (1) Let $\left(\mathcal{A}^{\prime \prime}, m_{0}\right)$ be a balanced multiarrangement. Then find a lower bound on

$$
\operatorname{MC}\left(\mathcal{A}^{\prime \prime}, m_{0}\right):=\min _{R(\overline{\mathcal{A}})=\left(\mathcal{A}^{\prime \prime}, m_{0}\right)}|\operatorname{ch}(\overline{\mathcal{A}})|
$$

(2) Let

$$
F_{h, n}:=\{\overline{\mathcal{A}} \text { : affine 2-arrangement } \mid \overline{\mathcal{A}} \text { is balanced, and } N R(\overline{\mathcal{A}})=(h, n)\} .
$$


Then find a lower bound on

$$
\operatorname{MC}(h, n):=\min _{\overline{\mathcal{A}} \in F_{h, n}}|\operatorname{ch}(\overline{\mathcal{A}})| .
$$

Problem 1.1 is interesting from viewpoints of both combinatorics and algebra in the following sense. First, Problem 1.1(2) is very easy to understand but not easy to answer. However, by using the formulation above, we can apply a rich theory of hyperplane arrangements to Problem 1.1. Note that

$$
M C(h, n)=\min _{N\left(\mathcal{A}^{\prime \prime}, m\right)=(h, n)} M C\left(\mathcal{A}^{\prime \prime}, m\right),
$$

and an answer to Problem 1.1(1) is given in [12]. Hence, we can use Problem 1.1(1) to study Problem 1.1(2). Then the relation to Yoshinaga's criterion appears, which is a deep result in the free arrangement theory. So at this point, a combinatorial problem is naturally related to an algebraic problem. Conversely, the relation between combinatorics and algebra (freeness) of arrangements have been studied by many mathematicians. Hence, an answer to Problem 1.1(2) can be a partial answer to this converse relation. We will give the definitions of the free arrangements in this section.

Before the main result, let us show an example to explain Problem 1.1.

Example 1.2 Put

$$
\left(\mathcal{A}^{\prime \prime}, m_{0}\right)=\left\{x_{1}^{2} x_{2}^{2}\left(x_{1}+x_{2}\right)^{2}\left(x_{1}-x_{2}\right)^{2}=0\right\}
$$

and consider Problem 1.1(1). Then it is easy to check that $M C\left(\mathcal{A}^{\prime \prime}, m_{0}\right)=25=$ $\left|\operatorname{ch}\left(\mathcal{A}_{1}\right)\right|$ with $\mathcal{A}_{1} \in F\left(\mathcal{A}^{\prime \prime}, m_{0}\right) \subset F_{4,8}$ defined by

$$
x_{1}\left(x_{1}-1\right) x_{2}\left(x_{2}-1\right)\left(x_{2}+x_{1}\right)\left(x_{2}+x_{1}-1\right)\left(x_{2}-x_{1}\right)\left(x_{2}-x_{1}-1\right)=0 .
$$

Next consider Problem 1.1(2). So we start from $N\left(\mathcal{A}^{\prime \prime}, m_{0}\right)=(4,8)$. Then $M C(4,8)$ is not 25 but $24=\left|\operatorname{ch}\left(\mathcal{A}_{2}\right)\right|$ with $\mathcal{A}_{2} \in F_{4,8}$ defined by

$$
x_{1}\left(x_{1}^{2}-1\right) x_{2}\left(x_{2}^{2}-1\right)\left(x_{1}^{2}-x_{2}^{2}\right)=0 .
$$

We will prove this by using Theorem 1.3 below.

For $a \in \mathbb{R},\lceil a\rceil:=\min \{b \in \mathbb{Z} \mid a \leq b\}$. Then we can state the first main result in this article.

Theorem 1.3 Let $2<h \leq n$ be integers, and $d:=\lceil(n-h+2) / 2\rceil$. Then

$$
M C(h, n) \geq(1+d)(1+n-d) .
$$

Hence, Theorem 1.3 shows that $M C(4,8)=24$ in Example 1.2. We prove Theorems 1.3 by using an algebraic method of 2-multiarrangements (Theorem 1.6), which is a new idea. Originally multiarrangements were introduced by Ziegler [14] to consider the Orlik's conjecture. Hence, only algebraic aspects of them have been studied, and there have been only few geometric results. However, Theorem 1.3 is clearly combinatorial and geometric. So Theorem 1.3 may be the starting point for us to apply the multiarrangement theory to the analysis of combinatorics and geometry 
of arrangements. Especially, it may work well to consider properties of a family of arrangements such as $R^{-1}\left(\mathcal{A}^{\prime \prime}, m_{0}\right)$ or $F_{h, n}$.

Now go back to Theorem 1.3. It is also an interesting problem to ask: if the equality holds in Theorem 1.3, then is the corresponding arrangement $\overline{\mathcal{A}}$ some special one? To answer this question, let us introduce some algebraic definitions of arrangements which we have already mentioned about before.

For a central multiarrangement $(\mathcal{A}, m)$, define the logarithmic derivation module $D(\mathcal{A}, m)$ by

$$
D(\mathcal{A}, m):=\left\{\theta \in \operatorname{Der}(S) \mid \theta\left(\alpha_{H}\right) \in S \cdot \alpha_{H}^{m(H)}(\forall H \in \mathcal{A})\right\} .
$$

$D(\mathcal{A}, m)$ is a reflexive module in general. When $D(\mathcal{A}, m)$ is a free module of rank $\ell$, we say that $(\mathcal{A}, m)$ is free, and for the homogeneous basis $\theta_{1}, \ldots, \theta_{\ell}$, we define

$$
\exp (\mathcal{A}, m):=\left(\operatorname{deg} \theta_{1}, \ldots, \operatorname{deg} \theta_{\ell}\right),
$$

where $\operatorname{deg} \theta:=\operatorname{deg} \theta(\alpha)$ for any $\alpha \in V^{*}$ such that $\theta(\alpha) \neq 0$. When $m \equiv 1$, the module $D(\mathcal{A}, 1)$ is also denoted by $D(\mathcal{A})$.

Actually, it is very difficult to determine whether a given arrangement is free. For example, the following Terao's conjecture is still unsolved:

Conjecture 1.4 (Terao) The freeness of a central arrangement $\mathcal{A}$ depends only on the combinatorics of $\mathcal{A}$, i.e., its intersection lattice $L(\mathcal{A})$.

For the definition of $L(\mathcal{A})$, see Sect. 1 . Now we can show that, if $\overline{\mathcal{A}}$ is the minimal chamber arrangement in the sense of Theorem 1.3 (i.e., it holds that $|\operatorname{ch}(\overline{\mathcal{A}})|=$ $\left.M C\left(\left|\mathcal{A}^{\prime \prime}\right|, \mid \overline{\mathcal{A}}\right) \mid\right)$, then it is free.

Theorem 1.5 In the notation above, if $|\operatorname{ch}(\overline{\mathcal{A}})|=(1+d)(1+n-d)$ for $\overline{\mathcal{A}} \in F_{h, n}$, then the coning $c \overline{\mathcal{A}}$ is a free arrangement.

For the definition of the coning, see Sect. 2. In fact, Theorem 1.5 is true for any base fields of characteristic zero in terms of the characteristic polynomial, see Theorem 4.5 for the generalized version. In this case, the freeness is determined by the combinatorics of arrangements.

For the proofs of Theorems 1.3 and 1.5, we use the theory of 2-multiarrangements, which is now closely related to the freeness of arrangements as follows. Recently, new freeness criterions were found by Yoshinaga in terms of restricted multiarrangements. Hence, it has become important to study the freeness of multiarrangements. In particular, by [12], to solve Conjecture 1.4 for 3-arrangements, the study of exponents of 2-multiarrangements is essential. Since 2-multiarrangements are free, we can always define the exponents. However, contrary to the simple arrangement case, the behavior of $\exp (\mathcal{A}, m)$ is complicated. See [11] for example. In this article we can give an upper bound on differences of exponents for balanced $(\mathcal{A}, m)$, which will play the key roles in the proofs of Theorems 1.3 and 1.5.

Theorem 1.6 Let $\mathcal{A}$ be a central 2-arrangement over a field of characteristic zero with $|\mathcal{A}|=h>2$. If $m: \mathcal{A} \rightarrow \mathbb{Z}_{>0}$ is balanced and $\exp (\mathcal{A}, m)=\left(d_{1}, d_{2}\right)$, then $\mid d_{1}-$ $d_{2} \mid \leq h-2$. 
For the proof, we use results in [3] and an affine connection $\nabla$, see Definition 3.1 for details.

Let us explain known results on 2-multiarrangements which are related to Theorem 1.6. Among related works, the most important result is proved by Wakefield and Yuzvinsky [11]. They proved that, roughly speaking, for $\exp (\mathcal{A}, m)=\left(d_{1}, d_{2}\right)$, generically $\left|d_{1}-d_{2}\right| \leq 1$. So exponents are very close in almost all cases. Theorem 1.6 concerns the exceptional case, i.e., the case where $\left|d_{1}-d_{2}\right|$ is the largest. Theorem 1.6 says that it can be at most $|\mathcal{A}|-2$. In the terminology of algebraic geometry, we can understand these two results as the splitting problem of rank two vector bundles on $\mathbf{P}^{2}$. Namely, [11] gives a result on generic splitting types, and Theorem 1.6 gives a bound of jumping types. Note that Theorem 1.6 does not hold over positive characteristic fields (see Remark 3.2).

The organization of this article is as follows. In Sect. 2 we introduce some definitions and results which will be used in this article. In Sect. 3 we prove Theorem 1.6. In Sect. 4 we prove Theorems 1.3 and 1.5. The latter will be proved in a more general setting as Theorem 4.5. Also we give several applications of Theorem 1.6 and examples of free 3-arrangements.

\section{Preliminaries}

In this section let us summarize the results and definitions that will be used in this article. For a general reference, see [7]. We use the notation of the introduction. For an affine $\ell$-arrangement $\overline{\mathcal{A}}$, the coning $c \overline{\mathcal{A}}$ of $\overline{\mathcal{A}}$ is an $(\ell+1)$-arrangement defined as follows. Let $Q \in \mathbb{K}\left[x_{1}, \ldots, x_{\ell}\right]$ be a defining polynomial of $\overline{\mathcal{A}}$, and $Q^{\prime} \in \mathbb{K}\left[x_{1}, \ldots, x_{\ell}, x_{\ell+1}\right]$ be the polynomial $Q$ homogenized. Then $c \overline{\mathcal{A}}$ is defined by $x_{\ell+1} Q^{\prime}=0$. The hyperplane $H_{0}=\left\{x_{\ell+1}=0\right\} \in c \overrightarrow{\mathcal{A}}$ is said to be the infinite hyperplane. For a simple arrangement $\overline{\mathcal{A}}$, define the intersection lattice $L(\overline{\mathcal{A}})$ by

$$
L(\overline{\mathcal{A}}):=\left\{\bigcap_{H \in \overline{\mathcal{B}}} H \mid \overline{\mathcal{B}} \subset \overline{\mathcal{A}}\right\} .
$$

This is a partially ordered set with the reverse inclusion order and the unique minimum element $V \in L(\overline{\mathcal{A}})$. The Möbius function $\mu$ on $L(\overline{\mathcal{A}})$ is defined by $\mu(V)=1$ and by

$$
\mu(X):=-\sum_{X \subsetneq Y} \mu(Y) \quad(X \in L(\overline{\mathcal{A}}) \backslash\{V\}) .
$$

The characteristic polynomial $\chi(\overline{\mathcal{A}}, t)$ is defined by

$$
\chi(\overline{\mathcal{A}}, t):=\sum_{X \in L(\overline{\mathcal{A}})} \mu(X) t^{\operatorname{dim} X} .
$$

The following is one of the most famous results in the arrangement theory, see [13] for the proof.

Theorem 2.1 (Zaslavsky) For a real arrangement $\overline{\mathcal{A}}$,

$$
|\operatorname{ch}(\overline{\mathcal{A}})|=|\chi(\overline{\mathcal{A}},-1)| .
$$


By Theorem 2.1 we can replace $|\operatorname{ch}(\overline{\mathcal{A}})|$ by $|\chi(\overline{\mathcal{A}},-1)|$, which allows us to describe Theorem 1.5 in terms of the characteristic polynomial over any field of characteristic zero. Then Theorem 1.5 can be generalized to the statement over any field of characteristic zero in Theorem 4.5 .

For a multiarrangement $(\mathcal{A}, m)$, recall $|m|=\sum_{H \in \mathcal{A}} m(H)$. The following is the most famous criterion for the freeness. For the proof, see [7, 8], and [14].

Theorem 2.2 (Saito's criterion) Let $\theta_{1}, \ldots, \theta_{\ell}$ be homogeneous derivations in $D(\mathcal{A}, m)$. Then $(\mathcal{A}, m)$ is free with basis $\left\{\theta_{1}, \ldots, \theta_{\ell}\right\}$ if and only if $\left\{\theta_{1}, \ldots, \theta_{\ell}\right\}$ is $S$-independent and $\sum_{i=1}^{\ell} \operatorname{deg} \theta_{i}=|m|$.

To use Yoshinaga's freeness criterion (Theorem 4.1), we must define the Ziegler restriction.

Definition 2.3 (Ziegler restriction) Let $\mathcal{A}$ be a central simple arrangement and fix $H_{0} \in \mathcal{A}$. The Ziegler restriction $\left(\mathcal{A}^{\prime \prime}, m_{0}\right)$ of $\mathcal{A}$ with respect to $H_{0}$ is defined by

$$
\begin{aligned}
\mathcal{A}^{\prime \prime} & :=\left\{H \cap H_{0} \mid H \in \mathcal{A} \backslash\left\{H_{0}\right\}\right\}, \\
m_{0}(K): & =\left|\left\{H \in \mathcal{A} \backslash\left\{H_{0}\right\} \mid H \cap H_{0}=K\right\}\right| \quad\left(K \in \mathcal{A}^{\prime \prime}\right) .
\end{aligned}
$$

Then for $D_{0}(\mathcal{A}):=\left\{\theta \in D(\mathcal{A}) \mid \theta\left(\alpha_{H_{0}}\right)=0\right\}$, the restriction map

$$
\pi: D_{0}(\mathcal{A}) \rightarrow D\left(\mathcal{A}^{\prime \prime}, m_{0}\right)
$$

is defined by $\pi(\theta):=\left.\theta\right|_{\alpha_{H_{0}}=0}$. See [14] for details.

Note that the Ziegler restriction is the same as $R(d \mathcal{A})\left(d \mathcal{A}:=\left.\mathcal{A} \backslash\left\{H_{0}\right\}\right|_{\alpha_{H_{0}}=1}\right)$ defined in the previous section. In the rest of this section assume that $\mathcal{A}$ is a central 2 -arrangement in $\mathbb{K}^{2}$. Let us recall results in [3]. Fix $\mathcal{A}$ and for a multiplicity $m$ : $\mathcal{A} \rightarrow \mathbb{Z}_{\geq 0}$, put $\exp (\mathcal{A}, m)=\left(d_{1}, d_{2}\right)$. Then the function $\Delta$ is defined by

$$
\Delta(m)=\left|d_{1}-d_{2}\right| .
$$

Let us consider all multiplicities $m$ on $\mathcal{A}$. In other words, we consider the multiplicity lattice $\Lambda$ and the subset $\Lambda^{\prime}$ defined by

$$
\begin{aligned}
& \Lambda:=\left\{m: \mathcal{A} \rightarrow \mathbb{Z}_{\geq 0}\right\}, \\
& \Lambda^{\prime}:=\{m \in \Lambda \mid \Delta(m) \neq 0\} .
\end{aligned}
$$

Then $\Delta$ is a function

$$
\Delta: \Lambda \rightarrow \mathbb{Z}_{\geq 0}
$$

Also, define

$$
\begin{aligned}
& \Lambda_{K}:=\left\{m \in \Lambda \mid m(K)>\sum_{H \in \mathcal{A} \backslash\{K\}} m(H)\right\} \quad(K \in \mathcal{A}), \\
& \Lambda_{0}:=\Lambda^{\prime} \cap\left(\Lambda \backslash \bigcup_{H \in \mathcal{A}} \Lambda_{H}\right) .
\end{aligned}
$$


Note that $\Lambda_{0}$ is denoted by $\Lambda_{\phi}^{\prime}$ in [3]. Define the distance $d\left(m, m^{\prime}\right)$ between $m, m^{\prime} \in$ $\Lambda$ by

$$
d\left(m, m^{\prime}\right):=\sum_{H \in \mathcal{A}}\left|m(H)-m^{\prime}(H)\right| .
$$

We say that two points $m, m^{\prime} \in \Lambda^{\prime}$ are connected if there exist points $m_{1}, \ldots, m_{n} \in \Lambda^{\prime}$ such that $d\left(m_{i}, m_{i+1}\right)=1(i=1, \ldots, n-1)$ and $m_{1}=m, m_{n}=m^{\prime}$. Then we can consider connected components of $\Lambda^{\prime}$. Note that, if we consider the Hasse graph structure of $\Lambda$ whose set of edges consists of $\left\{\left(m, m^{\prime}\right) \in \Lambda^{2} \mid d\left(m, m^{\prime}\right)=1\right\}$ and that of vertices $\Lambda$ itself, then the above connected component is the same as that in the induced Hasse graph structure. Then the following structure theorems hold for $\Lambda_{0}$.

Lemma 2.4 ([3], Lemmas 4.2 and 4.3) For $m_{1}, m_{2} \in \Lambda$ such that $d\left(m_{1}, m_{2}\right)=1$, let us put $\exp \left(\mathcal{A}, m_{i}\right)=\left(d_{1}^{i}, d_{2}^{i}\right)$ with $d_{1}^{i} \leq d_{2}^{i}(i=1,2)$. Then $\left|d_{j}^{1}-d_{j}^{2}\right|=1$ and $d_{k}^{1}=d_{k}^{2}$ for $\{j, k\}=\{1,2\}$. In particular, $\left|\Delta\left(m_{1}\right)-\Delta\left(m_{2}\right)\right|=1$.

Theorem 2.5 ([3], Theorem 3.2) Let $C \subset \Lambda_{0}$ be a maximal connected component of $\Lambda_{0}$. Then there exists a unique point $m \in C$, called the peak point of $C$, such that

$$
\Delta(m)>\Delta(\mu) \quad(\forall \mu \in C \backslash\{m\}) .
$$

Moreover,

$$
C=\left\{\mu \in \Lambda^{\prime} \mid d(m, \mu)<\Delta(m)\right\},
$$

and for $\mu \in \Lambda$ such that $d(\mu, m) \leq \Delta(m)$,

$$
\Delta(\mu)=\Delta(m)-d(m, \mu) .
$$

The maximal connected component of $\Lambda_{0}$ in Theorem 2.5 is just called a (finite) component, and $\Lambda_{K}$ an infinite component.

Remark 2.6 We always begin with a multiarrangement $(\mathcal{A}, m)$ such that $m: \mathcal{A} \rightarrow$ $\mathbb{Z}_{>0}$. However, in the arguments in the rest of this article, it often happens that a new multiplicity $m^{\prime}$ attains zero at some hyperplane $H \in \mathcal{A}$. However, as we have seen above, the theory in [3] is constructed in the multiplicity lattice $\Lambda=\left\{m: \mathcal{A} \rightarrow \mathbb{Z}_{\geq 0}\right\}$. Hence, there are no problems.

\section{The affine connection $\nabla$ and Theorem 1.6}

Let us start from the definition of $\nabla$.

Definition 3.1 For derivations $\theta$ and $\varphi=\sum_{i=1}^{\ell} f_{i} \partial_{x_{i}}$, define

$$
\nabla_{\theta} \varphi:=\sum_{i=1}^{\ell} \theta\left(f_{i}\right) \partial_{x_{i}} .
$$

Hence, $\nabla$ induces a map $\nabla: \operatorname{Der} S \times \operatorname{Der} S \rightarrow \operatorname{Der} S$. 
The main idea of the proof of Theorem 1.6 is to investigate the difference between $\theta$ and $\nabla_{\partial_{x_{i}}} \theta$, and combine it with the theory of an intersection lattice.

Proof of Theorem 1.6 Assume that $\Delta(m)>h-2$. We may assume that $\left\{x_{1} x_{2}=\right.$ $0\} \subset \mathcal{A}$ and take their dual basis $\partial_{x_{1}}, \partial_{x_{2}}$ for Der $S$. Let $C$ be a connected component of the multiplicity lattice of $\mathcal{A}$ such that $m \in C$. Since $\Delta$ attains the maximum value at the peak point of $C$, we may assume that $m$ is the peak point of $C$. Let $\theta$ be the lowest degree basis element for $D(\mathcal{A}, m)$ with $\operatorname{deg} \theta=d_{0}$. Then $\exp (\mathcal{A}, m)=$ $\left(d_{0}, d_{0}+\Delta(m)\right)$. If we put $n_{i}:=m\left(x_{i}=0\right)(i=1,2), n_{H}:=m(H)(H \in \mathcal{A})$, and

$$
\begin{aligned}
\theta\left(x_{j}\right) & =x_{j}^{n_{j}} f_{j} \quad(j=1,2), \\
\theta\left(\alpha_{H}\right) & =\alpha_{H}^{n_{H}} f_{H} \quad\left(H \in \mathcal{A} \backslash\left\{x_{1} x_{2}=0\right\}\right),
\end{aligned}
$$

then, for $\{i, j\}=\{1,2\}$,

$$
\begin{aligned}
\nabla_{\partial_{x_{i}}} \theta\left(x_{j}\right) & =x_{j}^{n_{j}} \partial_{x_{i}}\left(f_{j}\right), \\
\nabla_{\partial_{x_{i}}} \theta\left(x_{i}\right) & =x_{i}^{n_{i}-1}\left(n_{i} f_{i}+x_{i} \partial_{x_{i}}\left(f_{i}\right)\right), \\
\nabla_{\partial_{x_{i}}} \theta\left(\alpha_{H}\right) & =\alpha_{H}^{n_{H}-1}\left(n_{H} \partial_{x_{i}}\left(\alpha_{H}\right) f_{H}+\alpha_{H} \partial_{x_{i}}\left(f_{H}\right)\right) \quad\left(H \in \mathcal{A} \backslash\left\{x_{1} x_{2}=0\right\}\right) .
\end{aligned}
$$

Hence, the derivation $\nabla_{\partial_{x_{i}}} \theta$ belongs to $D\left(\mathcal{A}, m-m_{i}\right)$, where

$$
m_{i}(H)= \begin{cases}0 & \text { if } H=\left\{x_{j}=0\right\}, \\ 1 & \text { if } H \neq\left\{x_{j}=0\right\}\end{cases}
$$

with $\{i, j\}=\{1,2\}$. In other words, $\left(\mathcal{A}, m_{i}\right)$ is defined by

$$
x_{i}\left(\prod_{H \in \mathcal{A} \backslash\left\{x_{1} x_{2}=0\right\}} \alpha_{H}\right)=0 \quad(i=1,2) .
$$

Since $\left|m_{i}\right|=|\mathcal{A}|-1=h-1$ and $\Delta(m)>h-2$, it holds that $m-m_{i} \in C$ or on the boundary of $C$ (in other words, $d\left(m, m-m_{i}\right)=\Delta(m)$ ) for $i=1,2$.

Let us show that $\exp \left(\mathcal{A}, m-m_{i}\right)=\left(d_{0}, d_{0}+\Delta(m)-h+1\right)$. Let $m^{\prime}, m^{\prime \prime}$ be multiplicities between $m$ and $m-m_{i}$ such that $\left|m^{\prime}\right|=\left|m^{\prime \prime}\right|+1$. Then Lemma 2.4 and Theorem 2.5 show that $\Delta\left(m^{\prime}\right)=\Delta\left(m^{\prime \prime}\right)+1$, i.e., $\Delta$ decreases from $m$ to $m-$ $m_{i}$. Thus, Lemma 2.4 shows that $\exp \left(\mathcal{A}, m^{\prime}\right)=\left(d_{0}, d_{0}+\Delta(m)-d\left(m, m^{\prime}\right)\right)$, which implies $\exp \left(\mathcal{A}, m-m_{i}\right)=\left(d_{0}, d_{0}+\Delta(m)-h+1\right)$.

By the assumption, $d_{0} \leq d_{0}+\Delta(m)-h+1$. Since deg $\nabla_{\partial_{x_{i}}} \theta=d_{0}-1(i=1,2)$, it holds that

$$
\nabla_{\partial_{x_{i}}} \theta=0(i=1,2) .
$$

Since $\operatorname{char}(\mathbb{K})=0$, it holds that $\theta=a \partial_{x_{1}}+b \partial_{x_{2}}$ with $a, b \in \mathbb{K}$, which is a contradiction.

Remark 3.2 If $\operatorname{char}(\mathbb{K})=p>0$, then the statement in Theorem 1.6 does not hold. For example, assume that $p=2$ and consider the balanced multiarrangement $(\mathcal{A}, m)$ defined by

$$
x_{1}^{4} x_{2}^{4}\left(x_{1}+x_{2}\right)^{4}=0 .
$$


Then Theorem 2.2 shows that $x_{1}^{4} \partial_{x_{1}}+x_{2}^{4} \partial_{x_{2}}$ and $x_{1}^{8} \partial_{x_{1}}+x_{2}^{8} \partial_{x_{2}}$ form a basis for $D(\mathcal{A}, m)$. Hence, $\Delta(m)=4>|\mathcal{A}|-2=1$. See also [6].

On the other hand, the proof of Theorem 1.6 says that if $\operatorname{char}(\mathbb{K})=p>0, m \in \Lambda_{0}$ is a peak point, and $\Delta(m)>h-2$, then the degree of the lowest degree basis element for $D(\mathcal{A}, m)$ can be divided by $p$.

\section{Proofs of Theorems 1.3 and 1.5, and applications}

Before proving Theorems 1.3 and 1.5 let us recall one of Yoshinaga's freeness criterions.

Theorem 4.1 ([12], Theorem 3.2) Let $\mathcal{A}$ be a central 3-arrangement with the infinite hyperplane $H_{0} \in \mathcal{A}$. Let $\left(\mathcal{A}^{\prime \prime}, m_{0}\right)$ be the Ziegler restriction onto $H_{0}$ with $\exp \left(\mathcal{A}^{\prime \prime}, m_{0}\right)=\left(d_{1}, d_{2}\right)$. Also put

$$
\chi(\mathcal{A}, t)=(t-1)\left(t^{2}-c_{1} t+c_{2}\right) .
$$

Then, for the Ziegler restriction map

$$
\pi: D_{0}(\mathcal{A}) \rightarrow D\left(\mathcal{A}^{\prime \prime}, m_{0}\right),
$$

it holds that

$$
\operatorname{dim} \operatorname{coker} \pi=c_{2}-d_{1} d_{2} .
$$

Moreover, $\mathcal{A}$ is free with $\exp (\mathcal{A})=\left(1, d_{1}, d_{2}\right)$ if and only if $c_{2}=d_{1} d_{2}$.

Corollary 4.2 In the notation above, $c_{2} \geq d_{1} d_{2}$.

Corollary 4.2 follows immediately from Theorem 4.1, but it is very useful in the arguments below. Also, the following is an immediate and well-known consequence of Theorem 4.1, see [11] for example. Here we give a proof for completeness.

Proposition 4.3 Conjecture 1.4 is true for a 3-arrangement $\mathcal{A}$ such that its Ziegler restriction is not balanced.

Proof Fix an infinite hyperplane $H_{0} \in \mathcal{A}$ and put $\left(\mathcal{A}^{\prime \prime}, m_{0}\right)$ the Ziegler restriction of $\mathcal{A}$ onto $H_{0}$. By the assumption, $m_{0}(K)>\sum_{H \in \mathcal{A}^{\prime \prime} \backslash\{K\}} m_{0}(H)$ for some $K \in \mathcal{A}^{\prime \prime}$. We may assume that $\alpha_{K}=x_{1}$. Then

$$
\prod_{H \in \mathcal{A}^{\prime \prime} \backslash\{K\}} \alpha_{H}^{m_{0}(H)} \partial_{x_{2}}
$$

is the lowest degree basis element for $D\left(\mathcal{A}^{\prime \prime}, m_{0}\right)$. Hence, $\exp \left(\mathcal{A}^{\prime \prime}, m_{0}\right)=\left(m_{0}(K)\right.$, $\left.\left|m_{0}\right|-m_{0}(K)\right)$, and Theorem 4.1 says that $\mathcal{A}$ is free if $\chi(\mathcal{A}, t)=(t-1)(t-$ $\left.m_{0}(K)\right)\left(t-\left|m_{0}\right|+m_{0}(K)\right)$, which is combinatorial.

Now let us consider the balanced cases. Let us recall some notation. Let $\overline{\mathcal{A}}$ be an affine 2-arrangement over a field $\mathbb{K}$ of characteristic zero, and $c \overline{\mathcal{A}}$ its coning with 
the infinite hyperplane $H_{0} \in c \overline{\mathcal{A}}$. Put $|\overline{\mathcal{A}}|=n$ and $\chi(\overline{\mathcal{A}}, t)=\left(t^{2}-n t+c_{2}\right)$. Also, let $R(\overline{\mathcal{A}})=\left(\mathcal{A}^{\prime \prime}, m_{0}\right)$ be the Ziegler restriction of $c \overline{\mathcal{A}}$ onto $H_{0}$ with $\left|\mathcal{A}^{\prime \prime}\right|=h>2$. An integer $d$ is defined by $d:=\lceil(n-h+2) / 2\rceil$. We say that an affine simple 2arrangement $\overline{\mathcal{A}}$ is balanced if $m_{0}(K) \leq \sum_{H \in \mathcal{A}^{\prime \prime} \backslash\{K\}} m_{0}(H)$ for any $K \in \mathcal{A}^{\prime \prime}$. Now we can prove Theorems 1.3 and 1.5 .

Proof of Theorem 1.3 In this proof we assume that the base field $\mathbb{K}=\mathbb{R}$. By definition, it holds that $n=2 d+h-2$ or $n=2 d+h-3$. First, assume that $n=2 d+h-2$. Take an affine 2-arrangement $\overline{\mathcal{A}}$ such that $R(\overline{\mathcal{A}})=\left(\mathcal{A}^{\prime \prime}, m_{0}\right)$ and $N R(\overline{\mathcal{A}})=(h, 2 d+$ $h-2)$. Put $\exp \left(\mathcal{A}^{\prime \prime}, m_{0}\right)=\left(d_{1}, d_{2}\right)$. Note that $d_{1}+d_{2}=2 d+h-2=n$ by Theorem 2.2. Since $m_{0}$ is balanced, Theorem 1.6 implies that

$$
d_{1} d_{2} \geq d(d+h-2) \text {. }
$$

Also, Corollary 4.2 implies that

$$
c_{2} \geq d_{1} d_{2}
$$

Then Theorem 2.1 implies that

$$
\begin{aligned}
|\operatorname{ch}(\overline{\mathcal{A}})| & =|\chi(\overline{\mathcal{A}},-1)| \\
& =1+|\overline{\mathcal{A}}|+c_{2} \\
& \geq 1+(2 d+h-2)+d_{1} d_{2} \\
& \geq 1+(2 d+h-2)+d(d+h-2) \\
& =(1+d)(1+n-d),
\end{aligned}
$$

which completes the proof.

Second, assume that $n=2 d+h-3$. In this case, $n-(h-2)$ is an odd number. Hence, $\Delta\left(m_{0}\right)=\left|d_{1}-d_{2}\right| \leq h-3$ by Theorem 1.6. By the same arguments as above,

$$
c_{2} \geq d_{1} d_{2} \geq d(d+h-3),
$$

which, combined with $h=\left|\mathcal{A}^{\prime \prime}\right|>2$, completes the proof.

Remark 4.4 In Theorem 1.3 , if $\mathbb{R}$ is replaced by $\mathbb{C}$, then we obtain the lower bound on the total Betti number by the same proof.

Now we prove a generalized version of Theorem 1.5.

Theorem 4.5 In the notation above, if $\left(\mathcal{A}^{\prime \prime}, m_{0}\right)$ is balanced and $\chi(\overline{\mathcal{A}}, t)=(t-$ $d)(t-n+d)$ for $d=\lceil(n-h+2) / 2\rceil$, then $c \overline{\mathcal{A}}$ is free.

Note that every central 3-arrangement can be obtained as the coning of a certain affine 2-arrangement. Hence, Theorem 4.5 gives a sufficient combinatorial condition for certain 3-arrangements to be free.

Proof of Theorem 4.5 First, assume that $c_{2}=d(d+h-2)$ and $n=2 d+h-2$. Put $\exp \left(\mathcal{A}^{\prime \prime}, m_{0}\right)=\left(d_{1}, d_{2}\right)$. Note that $d_{1}+d_{2}=2 d+h-2=n$. Since $m_{0}$ is balanced, Theorem 1.6 implies that

$$
d_{1} d_{2} \geq d(d+h-2) \text {. }
$$


Also, Theorem 4.1 implies that

$$
c_{2}=d(d+h-2) \geq d_{1} d_{2} .
$$

If $\exp \left(\mathcal{A}^{\prime \prime}, m_{0}\right) \neq(d, d+h-2)$, then $d<\min \left\{d_{1}, d_{2}\right\}$ and $\max \left\{d_{1}, d_{2}\right\}<d+h-2$ by Theorem 1.6. Hence,

$$
c_{2}=d(d+h-2) \geq d_{1} d_{2}>d(d+h-2),
$$

which is a contradiction. Hence, $\exp \left(\mathcal{A}^{\prime \prime}, m_{0}\right)=(d, d+h-2)$, and Theorem 4.1 completes the proof.

Second, assume that $c_{2}=d(d+h-3)$ and $n=2 d+h-3=d_{1}+d_{2}$. In this case, $n-(h-2)$ is an odd number. Hence, $\Delta\left(m_{0}\right)=\left|d_{1}-d_{2}\right| \leq h-3$. By the same arguments as above,

$$
c_{2}=d(d+h-3) \geq d_{1} d_{2} \geq d(d+h-3),
$$

which, combined with $h=\left|\mathcal{A}^{\prime \prime}\right|>2$, completes the proof.

Proof of Theorem 1.5 Apply Theorems 2.1 and 4.5.

Remark 4.6 Theorems 4.1 and 4.5 show a close relation between free and minimal chamber arrangements as follows. First, $c \overline{\mathcal{A}}$ is free and $\overline{\mathcal{A}} \in R^{-1}\left(\mathcal{A}^{\prime \prime}, m_{0}\right)$ if and only if $\overline{\mathcal{A}}$ is a minimal chamber arrangement in $R^{-1}\left(\mathcal{A}^{\prime \prime}, m_{0}\right)$ in the sense of Problem 1.1(1). Second, if $\overline{\mathcal{A}} \in F_{h, n}$ is a minimal chamber arrangement in the sense of Problem 1.1(2), then $c \overline{\mathcal{A}}$ is free.

Hence, it is natural to study the same problems in higher-dimensional arrangements. For recent developments on this approach, see [2] and [5]. Namely, [2] gives a lower bound of chambers of affine tame arrangements the Ziegler restriction of which are the same tame multiarrangement, and [5] gives that of the coefficient of $t^{\ell-2}$ of $\chi(\mathcal{A}, t)$. Note that both bounds are given in terms of the characteristic polynomials of multiarrangements introduced in [4].

Theorem 1.6 has a lot of applications on the characteristic polynomials and freeness of 3-arrangements as follows.

Corollary 4.7 In the above notation, assume that $\overline{\mathcal{A}}$ is balanced and $\chi(\overline{\mathcal{A}}, t)=(t-$ $a)(t-b)$ with $a, b \in \mathbb{Z}, a \leq b$. Put $d=\lceil(n-h+2) / 2\rceil$. Then $d \leq a \leq b \leq n-d$.

Proof Since the proof is the same, we only prove the case where $n=2 d+h-2$. By Theorem 4.1 it holds that

$$
a b \geq d_{1} d_{2}
$$

with $\exp (\mathcal{A}, m)=\left(d_{1}, d_{2}\right)$. Note that $d_{1}+d_{2}=a+b$. By Theorem 1.6, $\left|d_{1}-d_{2}\right| \leq$ $h-2$. Hence, if $b-a>h-2$, then $a b-d_{1} d_{2}<0$, which is a contradiction.

Remark 4.8 These results say that if $\overline{\mathcal{A}}$ is balanced, then the characteristic polynomial $\chi(\overline{\mathcal{A}}, t)$ is irreducible over the integers or splits in the form above. Also, if $\chi(\overline{\mathcal{A}}, t)=(t-a)(t-b)$ such that $|a-b| \geq h-3$, then Conjecture 1.4 is true for these arrangements by Theorem 4.5 and Proposition 4.3. 
The following can be proved by using results in [10]. We give another proof here.

Corollary 4.9 Let $c \overline{\mathcal{A}}$ be a 3-arrangement with the infinite hyperplane $H_{0} \in c \overline{\mathcal{A}}$, and $\left(\mathcal{A}^{\prime \prime}, m_{0}\right)$ the Ziegler restriction of $c \overline{\mathcal{A}}$ onto $H_{0}$. Assume that $\left|\mathcal{A}^{\prime \prime}\right|=3$. Then the freeness of $c \overline{\mathcal{A}}$ depends only on $L(\overline{\mathcal{A}})$. In particular, so is that of the deformation of the Coxeter arrangement of type $A_{2}$.

Proof If $\left(\mathcal{A}^{\prime \prime}, m_{0}\right)$ is not balanced, then Proposition 4.3 completes the proof. Assume that $\left(\mathcal{A}^{\prime \prime}, m_{0}\right)$ is a balanced Coxeter multiarrangement of type $A_{2}$. Since $\left|\mathcal{A}^{\prime \prime}\right|-2=1$, Theorem 4.5 shows that $c \overline{\mathcal{A}}$ is free if and only if $\chi(\overline{\mathcal{A}}, t)=(t-a)^{2}$ or $\chi(\overline{\mathcal{A}}, t)=$ $(t-a)(t-a-1)$, which completes the proof.

Example 4.10 Let $\mathcal{A}$ be the Coxeter arrangement of type $B_{2}$, and $\overline{\mathcal{A}}$ its deformation as in [1]. The freenesses of such deformations have not yet been classified, nor have been the exponents of the multiarrangements $(\mathcal{A}, m)$. Some of the freenesses of $c \overline{\mathcal{A}}$ were classified in Propositions 2.3 and 2.4 in [1]. They were proved by using the addition theorem in [9]. If we use Theorem 4.5, then the explicit formula of the Poincaré polynomial ([1], Lemma 2.1) is enough to show the freeness of some cases. In other words, Theorem 4.7 says that if $\overline{\mathcal{A}}$ is balanced and $\chi(\overline{\mathcal{A}}, t)$ splits, then it is of one of the form

$$
\begin{aligned}
& \chi(\overline{\mathcal{A}}, t)=(t-d)^{2}, \\
& \chi(\overline{\mathcal{A}}, t)=(t-d)(t-d+1), \text { or } \\
& \chi(\overline{\mathcal{A}}, t)=(t-d)(t-d+2) .
\end{aligned}
$$

Also, Theorem 4.5 says that $c \overline{\mathcal{A}}$ is free if $\chi(\overline{\mathcal{A}}, t)$ splits into the form of the second and third types in the above equations.

More generally, when $|\mathcal{A}|=4$, the following holds.

Corollary 4.11 Let $\mathcal{A}$ be a central 3-arrangement such that $\left|\mathcal{A} \cap H_{0}\right|=4$ for some $H_{0} \in \mathcal{A}$. If $|\mathcal{A}|$ is even, then the freeness of $\mathcal{A}$ depends only on $L(\mathcal{A})$.

Proof If the deconing $d \mathcal{A}:=\left.\left(\mathcal{A} \backslash\left\{H_{0}\right\}\right)\right|_{\alpha_{H_{0}}=1}$ (a converse operation of the coning) is not balanced, then Proposition 4.3 completes the proof. Assume that $d \mathcal{A}$ is balanced. In this assumption, the splitting type of the characteristic polynomial is always

$$
\chi(\mathcal{A}, t)=(t-1)(t-d)(t-d-1)
$$

by Corollary 4.7. Hence, Theorem 4.5 completes the proof.

Remark 4.12 Let us consider Problem 1.1(2) when an affine 2-arrangement $\overline{\mathcal{A}}$ is not balanced. Put $N R(\overline{\mathcal{A}})=(h, n)$ and suppose that $R^{-1}\left(N^{-1}(h, n)\right)$ contains a nonbalanced $\overline{\mathcal{A}}$. We may assume that $\{x=0\} \in \overline{\mathcal{A}}$. Then consider $\overline{\mathcal{A}}$ defined by

$$
\begin{aligned}
x & =0,1, \ldots, n-h, \\
\alpha_{i} & =0 \quad(i=1, \ldots, h-1),
\end{aligned}
$$


Fig. 1 Examples of a balanced and nonbalanced arrangements
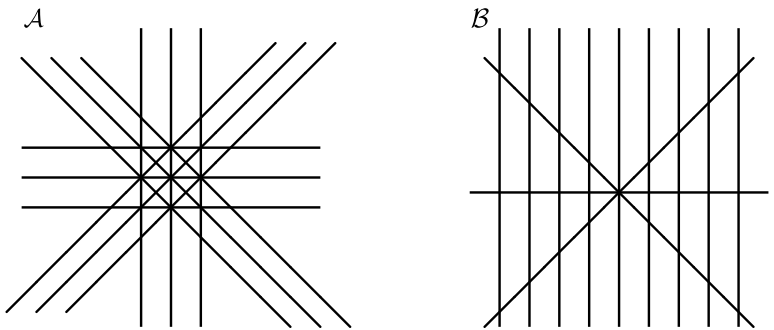

where any two of $x, \alpha_{1}, \ldots, \alpha_{h-1}$ define different lines. Then

$$
|\operatorname{ch}(\overline{\mathcal{A}})|=(2+n-h) h=n h-h^{2}+2 h,
$$

which can be smaller than the lower bound in Theorem 1.3. Namely, by using $n=$ $2 d+h-2$ or $n=2 d+h-3$, computations show that $h(n-h+2)<(1+d)(1+$ $n-d$ ) if and only if

$$
\begin{array}{ll}
n>3 h-4 & (n \text { is even }), \\
n>3 h-5 & (n \text { is odd }) .
\end{array}
$$

For example, there are two arrangements $\mathcal{A}$ and $\mathcal{B}$ in Fig. 1 with directions $h=4$ and the number of lines $n=12$. Then the lower bound of chambers by Theorem 1.3 is 48. Since $|\operatorname{ch}(\mathcal{A})|=48$ and $\mathcal{A}$ is balanced, $\mathcal{A}$ is the minimal chamber arrangement in the sense of Problem 1.1(2). Since $\mathcal{B}$ is not balanced, this bound does not work. In fact, $|\operatorname{ch}(\mathcal{B})|=40$, which is nothing but the above lower bound $h(n-h+2)=$ $4 \times 10=40$.

Acknowledgements The author thanks Yasuhide Numata for reading the first draft of this article and pointing out a mistake. The author is grateful to the referees for the careful reading and a lot of useful comments on this article. This work is supported by JSPS Grants-in-Aid for Young Scientists (B) No. 21740014.

\section{References}

1. Abe, T.: The stability of the family of $B_{2}$-type arrangements. Commun. Algebra 37(4), 1193-1215 (2009)

2. Abe, T.: Characteristic polynomials, $\eta$-complexes and freeness of tame arrangements. Michigan Math. J. (2012, to appear). arXiv:1109.1042v1

3. Abe, T., Numata, Y.: Exponents of 2-multiarrangements and multiplicity lattices. J. Algebr. Comb. 35(1), 1-17 (2012)

4. Abe, T., Terao, H., Wakefield, M.: The characteristic polynomial of a multiarrangement. Adv. Math. 215, 825-838 (2007)

5. Abe, T., Yoshinaga, M.: Free arrangements and coefficients of characteristic polynomials. arXiv:1109. $0668 \mathrm{v} 1$

6. Numata, Y.: An algorithm to construct a basis for the module of logarithmic vector fields. arXiv:0707. 0004

7. Orlik, P., Terao, H.: Arrangements of Hyperplanes. Grundlehren der Mathematischen Wissenschaften, vol. 300. Springer, Berlin (1992)

8. Saito, K.: Theory of logarithmic differential forms and logarithmic vector fields. J. Fac. Sci., Univ. Tokyo, Sect. 1A, Math. 27, 265-291 (1980) 
9. Terao, H.: Arrangements of hyperplanes and their freeness I, II. J. Fac. Sci., Univ. Tokyo, Sect. 1A, Math. 27, 293-320 (1980)

10. Wakamiko, A.: On the exponents of 2-multiarrangements. Tokyo J. Math. 30(1), 99-116 (2007)

11. Wakefield, M., Yuzvinsky, S.: Derivations of an effective divisor on the complex projective line. Trans. Am. Math. Soc. 359(9), 4389-4403 (2007)

12. Yoshinaga, M.: On the freeness of 3-arrangements. Bull. Lond. Math. Soc. 37(1), 126-134 (2005)

13. Zaslavsky, T.: Facing up to arrangements: face-count formulas for partitions of spaces by hyperplanes. Mem. Am. Math. Soc. 154 (1975)

14. Ziegler, G.M.: Multiarrangements of hyperplanes and their freeness. In: Singularities, Iowa City, IA, 1986. Contemp. Math., vol. 90, pp. 345-359. Amer. Math. Soc., Providence (1989) 